\title{
Subjetividades y actuación pedagógica del personal docente de educación física en contextos de diversidad cultural en la región de Puno-Perú
}

\author{
Subjectivities and pedagogical intervention of Physical Education teachers in contexts of \\ cultural diversity in the Puno-Peru region
}

\section{Subjetividades e atuação pedagógica do corpo docente de educação física em contextos de diversidade cultural na região de Puno-Peru}

Yanet Amanda Maquera-Maquera

Universidad Nacional del Altiplano

Puno, Perú

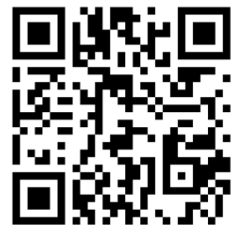

ymaquera@unap.edu.pe

https://orcid.org/0000-0002-2492-6428

Saúl Bermejo-Paredes

Universidad Nacional del Altiplano

Puno, Perú

sbermejo@unap.edu.pe

iD https://orcid.org/0000-0001-9885-7974

Recibido • Received • Recebido: 26 / 06 / 2019

Corregido • Revised • Revisado: 25 / 01 / 2021

Aceptado • Accepted • Aprovado: 01 / 03 / 2021

\begin{abstract}
Resumen: La nueva coyuntura en la que nos encontramos ha cambiado profundamente las maneras de enseñar y aprender la educación física (EF). La relevancia del desempeño docente en la complejidad de la enseñanza está siendo cuestionada y existe una tensión permanente entre la calidad y la pertinencia de las prácticas pedagógicas en EF. El estudio tuvo por objetivo comprender la naturaleza de las subjetividades y la actuación pedagógica del personal docente de EF, en contextos de diversidad cultural, desde la metodología cualitativa-hermenéutica. Se utilizó la técnica narrativa y la entrevista en profundidad con el personal docente de la Escuela Profesional de Educación Física, Universidad Nacional del Altiplano (UNA) y del Instituto de Educación Superior Pedagógico Público Educación Física-Lampa, participantes del Programa de Fortalecimiento de Capacidades-Ministerio de Educación del Perú 2019, en convenio con la UNA-Puno. Al haberse establecido 03 categorías de análisis: autorreflexión de la práctica pedagógica, creencias-actuaciones pedagógicas y afrontamiento de la diversidad cultural, los resultados alcanzados a través del análisis de contenido posibilitan comprender que las subjetividades del personal docente de EF definen los sentidos otorgados a su actuación pedagógica que, por lo general, se caracterizan por el desencuentro entre los saberes experienciales, pedagógicos y de enseñanza, poco pertinentes a un contexto de diversidad cultural predominante en la región Puno. Existe una ruptura con lo que se es y con lo que se hace realmente, por lo que se requiere redefinir la actuación pedagógica del personal docente de EF e incorporar sus creencias, pensamientos y actitudes desde la singularidad histórico-cultural contextualizada.
\end{abstract}


http://doi.org/10.15359/ree.25-2.9

http://www.una.ac.cr/educare

educare@una.ac.cr

Palabras claves: Subjetividad docente; diversidad cultural; enseñanza educación física; quehacer pedagógico.

\begin{abstract}
The new circumstances we live in have profoundly changed the ways of teaching and learning Physical Education (PE).The importance of a professor's performance in the complexity of teaching is being questioned, and there is a permanent tension between the quality and relevance of pedagogical practices in PE. From a qualitative-hermeneutic methodology, this research aimed to understand the nature of the subjectivities and the pedagogical performance of PE teachers in contexts of cultural diversity. The narrative technique and in-depth interview were used with the teaching staff of the Professional School of Physical Education, at the National University of the Altiplano (UNA), and the Institute of Higher Education Pedagogical Public Physical Education-Lampa; the teachers were participating in the Program of Strengthening of Capacities-Ministry of Education of Peru 2019, in agreement with UNA-Puno. The following three categories were established for the analysis: self-reflection of pedagogical practice, pedagogical beliefs and actions, and coping with cultural diversity. The results achieved through the content analysis make it possible to understand that the subjectivities of PE teaching staff define the meanings given to their pedagogical performance. These meanings are generally characterized by the disagreement between experiential, pedagogical, and teaching knowledge, which are not very relevant to a context of predominant cultural diversity in the Puno region. There is a gap between what is and what is really done, so it is necessary to redefine PE teachers' pedagogical performance of and incorporate their beliefs, thoughts, and attitudes from the contextualized historical-cultural singularity.
\end{abstract}

Keywords: Teaching subjectivity; cultural diversity; teaching physical education; pedagogical work.

Resumo: A nova situação em que nos encontramos mudou profundamente as formas de ensinar e aprender Educação Física (EF). Questiona-se a relevância da atuação docente na complexidade do ensino, onde existe uma tensão permanente entre a qualidade e a relevância das práticas pedagógicas em EF. O objetivo do estudo foi compreender a natureza das subjetividades e da atuação pedagógica de professores de EF, em contextos de diversidade cultural, a partir da metodologia qualitativohermenêutica. A técnica utilizada foi a narrativa e entrevista em profundidade com o corpo docente da Escola Profissional de Educação Física, da Universidade Nacional do Altiplano (UNA) e o Instituto de Educação Superior Pedagógica de Educação Física Pública-Lampa, participantes do Programa de Fortalecimento de Capacidades-Ministério da Educação do Peru 2019, em convênio com UNAPuno. Foram estabelecidas 03 categorias de análise: autorreflexão da prática pedagógica, crençasações pedagógicas e enfrentamento da diversidade cultural; os resultados alcançados por meio da análise de conteúdo permitem compreender que as subjetividades dos docentes de EF definem os significados atribuídos à sua atuação pedagógica, que geralmente se caracterizam pela discordância entre saberes vivenciais, pedagógicos e docentes, os quais são pouco relevantes. um contexto de diversidade cultural predominante na região de Puno. Há uma ruptura com o ser e o fazer, exigindo redefinir a atuação pedagógica do professor de EF incorporando suas crenças, pensamentos e atitudes a partir da singularidade histórico-cultural contextualizada.

Palavras-chave: Subjetividade docente; diversidade cultural; ensino de educação física; trabalho pedagógico.

Yanet Amanda Maquera-Maquera y Saúl Bermejo-Paredes

Los artículos de la Revista Electrónica Educare del Centro de Investigación y Docencia en Educación de la Universidad Nacional, Costa Rica, se comparten bajo términos de la Licencia Creative Commons: Reconocimiento, № Comercial, Sin Obra Derivada 3.0 Costa Rica. Las autorizaciones adicionales a las aquí delimitadas se pueden obtener en el correo: educare@una.cr 
http://doi.org/10.15359/ree.25-2.9

\section{Introducción}

Las universidades y los institutos de educación superior peruanas, ante las exigencias y políticas del contexto hegemónico mundial, signado por situaciones dinámicas, nuevas e impredecibles; la certificación y la calidad en la formación de profesionales; la emergencia de enfoques y regulación de las formas de organización académica e institucional, así como la estandarización de las prácticas pedagógicas de enseñanza y aprendizaje, han generado, en el personal docente de educación física, una fuerte tensión que pone en crisis su subjetividad respecto a su desempeño pedagógico, su estatus profesional, entre lo que realmente hace y lo que el sistema y las políticas de educación superior le exigen.

El estudio de la subjetividad docente en contextos de diversidad cultural, a partir del despliegue individual y disciplinar de experiencias y conocimientos en el aula, es poco conocido, excepcionalmente; algunas investigaciones han permitido aproximarse a temáticas como las creencias, percepciones, interacciones, prácticas simbólicas, imaginarios culturales, y los marcos teórico-interpretativos que definen a los individuos en relación con sus propias concepciones de mundo y complejos entramados de significaciones que va a constituir, finalmente, subjetividades fragmentadas y múltiples (Patiño-Garzón y Rojas-Betancur, 2009). No obstante, el abordaje de la subjetividad desde la perspectiva histórico-cultural es la más pertinente a nuestra realidad educativa y problemática planteada que, según González Rey (2002), viene a constituir:

[La] unidad de los procesos simbólicos y las emociones, donde la emergencia de uno de ellos evoca al otro sin convertirse en su causa, formando verdaderas cadenas con formas muy diversas de expresión según el contexto en que la persona está implicada. (p. 312)

Comprender las actuaciones pedagógicas a partir de la subjetivad del personal docente de educación física, en un contexto de diversidad cultural (región Puno-Perú), es muy relevante y complejo. Las actuaciones pedagógicas en este estudio son entendidas como las actividades diarias de carácter procesual desarrolladas en el aula, orientadas por el currículo, que prestan mayor atención a las interacciones docente-estudiante, las condiciones y el contexto (Castellanos Galindo y Yaya Escobar, 2013; Cerdá Michael, 2001). Por diversidad cultural se concibe un territorio histórico-cultural marcado, mayoritariamente, por la presencia de culturas ancestrales como el aimara y el quechua, donde el elemento central de la estructuración social e intersubjetiva descansa en la colonialidad del poder, sustentada en la idea de"raza" y el complejo ideológico del racismo, como formas efectivas de dominación social, material e intersubjetiva (Quijano, 2000). 
http://doi.org/10.15359/ree.25-2.9

http://www.una.ac.cr/educare

educare@una.ac.cr

En la generalidad de los casos, las subjetividades o creencias personales, el conocimiento didáctico del contenido, estructuras de pensamiento y las formas de actuar, configuran o distinguen con un sello particular las prácticas pedagógicas del personal docente, así Hernández-Álvarez et al. (2010) señalan, categóricamente, que el desempeño y actuar de docentes de EF está condicionado por las creencias y concepciones que el mismo grupo profesional tiene respecto a su rol. En esta perspectiva de pensamiento y la problemática expuesta, se ha planteado, como objetivo principal de estudio, comprender la naturaleza de las representaciones subjetivas y la actuación pedagógica en el personal docente de educación física de la Escuela Profesional de Educación Física-Universidad Nacional del Altiplano (EPEF-UNA Puno) y el Instituto de Educación Superior Pedagógico Público Educación Física-Lampa (ISPPEF-Lampa), de la región Puno-Perú, en contextos de diversidad cultural aimara, quechua y español.

El creciente interés por asegurar una educación inclusiva, de calidad y equidad, en contextos históricamente excluidos, es aún una utopía; pero, a la vez, es un reto permanente para poner en agenda en los círculos decisorios, académicos y políticos, con el propósito de revertir una deuda histórica, a favor de la democracia, la justicia social, la inclusión, los derechos humanos, la dignidad, la paz, la seguridad, la diversidad cultural-lingüística y un auténtico desarrollo humano sostenible y armónico. Los resultados del estudio deben coadyuvar a la transformación de las estructuras organizacionales-institucionales de los centros superiores de formación docente; la construcción de las políticas de desarrollo institucional para incorporar, explícitamente, las subjetividades y experiencias pedagógicas del personal docente de EF, para el fortalecimiento de las capacidades profesionales y desarrollo pedagógico del área curricular de educación física.

\section{Metodología}

La investigación se define como cualitativa-hermenéutica, sigue un procedimiento inductivo para comprender el sentido otorgado a las narraciones o experiencias de vida humana, en su propio contexto (Corbetta, 2003; Martínez, 1999). Las técnicas utilizadas para el recoger la información, con información consentida, correspondieron a la entrevista en profundidad y narrativa, por permitir encuentros cara a cara entre quien investiga y sus informantes, para llegar a la información requerida según los propósitos del estudio y poder interpretar los hechos y acciones narrados por el profesorado a partir de sus propias vivencias (Bolívar Botía et al., 2001; Flick, 2007). Las narraciones consistieron en el relato escrito individual referido a sus conocimientos, creencias y experiencias de actuación pedagógica. Se generaron a través de las siguientes interrogantes: ¿En qué consistió el problema o desafío que afrontaste en dicha práctica?, ¿qué decisiones pedagógicas tomaste para resolver el 
http://doi.org/10.15359/ree.25-2.9

problema o desafío?, ¿qué conocimientos, creencias o teorías pedagógicas influyeron en las decisiones que tomaste?, ¿qué estrategias utilizaste?, ¿cuáles fueron las consecuencias de las decisiones pedagógicas tomadas? Estas mismas fueron elaboradas y validadas por especialistas y docentes a cargo de la formación del Programa de Fortalecimiento de Capacidades (PFC) para docentes de educación superior pedagógica, implementada por el Ministerio de Educación del Perú, durante el 2019. Estas preguntas se administraron luego de concluir el primer módulo del PFC (enfoques y epistemología de la educación), durante los talleres presenciales ejecutados con el personal docente participante y la autenticidad de las narraciones estuvo al cuidado del segundo autor del presente estudio, que formó parte del programa como docente formador. La entrevista en profundidad estuvo dirigida básicamente a recabar información respecto a las categorías de análisis: autorreflexión sobre la práctica pedagógica y afrontamiento de la diversidad cultural, se realizó en espacios académicos y contextos situacionales de interacción dialógica y de confianza mutua entre los sujetos entrevistadores y los sujetos entrevistados. El guion elaborado para recabar datos referidos a la autorreflexión sobre la práctica pedagógica consistió en las siguientes preguntas: ¿Qué acciones realizo en los cursos que dicto? ¿Por qué? ¿Qué actividades me gustaría realizar en los cursos que dicto? ¿Por qué no las he realizado hasta ahora? ¿Qué oportunidades me brindan mis cursos para poder realizarlas?

\section{Participantes y contexto}

La selección de docentes no obedeció a contar con una representación estadística, sino consistió en cubrir sistemáticamente información suficiente según el objetivo del estudio y tópicos del guion de las entrevistas. Aceptaron participar del estudio, de manera voluntaria y con consentimiento informado 08 docentes de EF, que representan el 30\% del total de personal docente perteneciente a la Escuela Profesional de Educación FísicaUniversidad Nacional del Altiplano (EPEF-UNA Puno) y al Instituto de Educación Superior Pedagógico Público Educación Física-Lampa (ISPPEF-Lampa). Ambas instituciones están regidas por el Estado peruano a través del Ministerio de Educación y son las únicas constituidas para la formación inicial de docentes en educación física en la región de Puno, ubicada entre las cordilleras del extremo Sur-Este del territorio peruano, con una población mayoritariamente de origen indígena aimara y quechua. La ejecución de la investigación comprendió de diciembre 2018 a mayo de 2019, en el marco del Programa de Fortalecimiento de Capacidades (PFC), implementada por el Ministerio de Educación del Perú (ISPPEF-Lampa), en convenio con la Universidad Nacional del Altiplano de Puno-Perú. Las características de quienes participaron pueden contemplarse en la Tabla 1. 
http://doi.org/10.15359/ree.25-2.9

http://www.una.ac.cr/educare

educare@una.ac.cr

Tabla 1: Características de docentes participantes

Escuela Profesional EF-UNA Puno

a. 02 docentes mujeres responsables del desarrollo de asignaturas de formación básica y especializada del currículo de la EPEF-UNA Puno, registran 08 años promedio de ejercicio profesional, ostentan título profesional de Licenciado en Educación Física y los grados académicos de magíster y doctor en educación. Para el análisis de datos fueron registradas con los códigos P-1 y P-2.

b. 02 docentes varones responsables del desarrollo de asignaturas de formación básica y especializada del currículo de la EPEF-UNA Puno, registran 14 años promedio de ejercicio profesional, ostentan título profesional de Licenciado en Educación Física y los grados académicos de magíster y doctor en educación. Códigos asignados para el análisis de datos: P-3 y P-4.

Total docentes participantes: 04
Instituto SPPEF-Lampa

a. 01 docente mujer responsable del desarrollo de asignaturas de formación básica y especializada del currículo ISPPEF-Lampa, registra 29 años de ejercicio profesional, ostenta título profesional pedagógico y los grados académicos de magíster y doctor en educación. Código asignado para el análisis de datos: L-1.

b. 03 docentes varones con título profesional de Profesor de Educación Física, responsables del desarrollo de asignaturas de formación básica y especializada del currículo ISPPEF-Lampa, registran 26 años promedio de ejercicio profesional, 01 de ellos ha concluido estudios de postgrado. Códigos asignados para el análisis de datos: L-2, L-3 y L-4.

Nota: Elaboración propia.

\section{Procedimiento para recojo de información}

Entre los mecanismos implementados para el recojo de información destacaron: lograr el consentimiento de la Coordinación Académica del PFC para desarrollar el trabajo de investigación; selección de informantes que aceptaron participar del estudio; informar al personal docente involucrado respecto a la finalidad de las entrevistas; contar con un guion temático de preguntas; concertar ambiente y tiempo a dedicación exclusiva para la entrevista; prever y disponer de medios necesarios para asegurar el éxito en la recogida de información; iniciar la entrevista de manera dinámica y lograr la aceptación de la grabación; controlar los tiempos y evitar extravíos o dispersiones en los sujetos entrevistados; mantener un diálogo asertivo y abierto; en el caso de las narrativas escritas, fijar un tiempo de entrega; finalizar la entrevista cordialmente e iniciar la transcripción, anotar algunos detalles como frases, repeticiones, omisiones, titubeos, gesticulaciones y formas de expresarse.

\section{Procedimiento análisis de datos}

Los datos fueron analizados mediante el análisis de contenido, entendido como una técnica de interpretación de registro de datos de manera sistemática y exhaustiva para comprender los 
http://doi.org/10.15359/ree.25-2.9

http://www.una.ac.cr/educare educare@una.ac.cr

diversos aspectos y fenómenos de la vida social (Andréu Abela, 2002; Krippendorff, 1990). La aplicación de la técnica del análisis de contenido contempló 03 procesos: determinación objeto de análisis, definición de categorías/codificación y análisis/interpretación, según el detalle contenido en la Tabla 2.

Tabla 2: Procesos implementados para el análisis de contenido

\begin{tabular}{|c|c|c|}
\hline Determinación objeto de análisis & Definición categorías / Codificación & Análisis / Interpretación \\
\hline $\begin{array}{l}\text { - Al inicio se examina y ordena } \\
\text { cuidadosamente cada } \\
\text { transcripción, cotejando con las } \\
\text { observaciones y comentarios, } \\
\text { en camino a la construcción de } \\
\text { conceptos e interpretaciones. } \\
\text { - Determinación del problema } \\
\text { (tiempo, espacio, personas y } \\
\text { contexto) y objetivo del estudio } \\
\text { (objeto de análisis). } \\
\text { - Seleccionar y analizar el marco } \\
\text { teórico-conceptual existente } \\
\text { para entender mejor el problema } \\
\text { y el procesamiento de los datos. }\end{array}$ & $\begin{array}{l}\text { - Las categorías de análisis fueron } \\
\text { definidas considerando la } \\
\text { opinión experta y especializada } \\
\text { del PFC y la intervención de } \\
\text { participantes, a partir de la } \\
\text { fiabilidad y validez del núcleo de } \\
\text { ideas y significados referidos a la } \\
\text { temática en estudio. } \\
\text { - Categorías del estudio: } \\
\text { autorreflexión de la práctica } \\
\text { pedagógica, creencias / actuación } \\
\text { pedagógica y afrontamiento de la } \\
\text { diversidad cultural. } \\
\text { - El proceso de codificación } \\
\text { consistió en: recopilar y contrastar } \\
\text { información por categorías de } \\
\text { análisis, con asignación de una } \\
\text { unidad de registro o código para } \\
\text { sistematizar e identificar contextos, } \\
\text { categorías y participantes. } \\
\text { - Los códigos adjudicados a las } \\
\text { unidades de datos fueron: P } \\
\text { = Puno (marca identificatoria } \\
\text { docentes EPEF-UNA-Puno); L = } \\
\text { Lampa (marca asignada a docentes } \\
\text { ISPPEF-Lampa); } 1,2,3 \text { y } 4 \text { para } \\
\text { identificar docentes participantes. }\end{array}$ & $\begin{array}{l}\text { - Análisis o evaluación exhaustiva } \\
\text { de la información seleccionada } \\
\text { en términos de inclusión o } \\
\text { exclusión acorde a la temática } \\
\text { y objetivo del estudio, en } \\
\text { perspectiva a la construcción de } \\
\text { los sentidos o interpretaciones. } \\
\text { - La interpretación se sustentó } \\
\text { en el proceso de análisis } \\
\text { reinterpretativo (interpretación } \\
\text { de la interpretación), en dos } \\
\text { fases: la primera, sistematizó } \\
\text { y organizó los contenidos por } \\
\text { categorías para facilitar la síntesis } \\
\text { y el análisis del corpus, desde } \\
\text { la perspectiva del personal } \\
\text { docente que proporcionó } \\
\text { por escrito y a través de las } \\
\text { entrevistas en profundidad y las } \\
\text { narraciones escritas; la segunda } \\
\text { fue para el análisis de contenido, } \\
\text { comprensión e interpretación- } \\
\text { reinterpretación de las } \\
\text { subjetividades que prevalecen } \\
\text { en el personal docente durante la } \\
\text { puesta en acción de los procesos } \\
\text { pedagógicos en el área de } \\
\text { educación física. }\end{array}$ \\
\hline
\end{tabular}

Nota: Elaboración propia. 
http://doi.org/10.15359/ree.25-2.9

http://www.una.ac.cr/educare

educare@una.ac.cr

\section{Resultados, análisis y discusión}

\section{Autorreflexión de la práctica pedagógica}

El personal docente involucrado en este estudio estuvo dispuesto a problematizar, explicitar y reflexionar sobre sus experiencias pedagógicas, en relación con su trabajo profesional: ...tengo muchas experiencias que han ocurrido a lo largo de mi actividad docente. $A$ veces los comparto con los amigos más próximos, y nadies más. Siento que es de mi incumbencia y no parece interesarle a nadie. Pero, si usted tiene un verdadero interés por saber mis experiencias desarrolladas en la enseñanza durante mi labor de docente, no tengo ningún inconveniente... (L1). Esta misma predisposición y voluntad se advierte en cada docente en general, aspecto que favoreció la elaboración de los relatos individuales, referidos a la descripción de las experiencias pedagógicas vividas.

La autorreflexión de la práctica pedagógica por el personal docente de EF es comprendida como las percepciones que tienen de sí mismo, conformada por el cúmulo de conocimientos, experiencias, valoraciones construidas en diversos contextos y momentos, en cuanto al ejercicio profesional de la función docente: ... aún no es suficiente lo que sé, tengo experiencia en educación básica pero la educación superior es distinta $(P-2) ;$... la mayoría estamos dedicados al desarrollo de nuestra carga horaria, nuestras asignaturas, me gustaría especializarme más o experimentar y desarrollar investigaciones, pero hay muchas limitaciones...(P-4); se caracterizan por enfatizar la tarea docente dirigida al desarrollo curricular de las asignaturas y por las aspiraciones profesionales o metas por cubrir.

La autorreflexión sobre las prácticas pedagógicas, mediante un análisis constructivo puede constituirse, según las narraciones que se presentan, en una fuente compartida para la mejora de la práctica profesional, en una posibilidad para encarar el trabajo cotidiano, desarrollar una revisión de conocimientos, sentimientos y percepciones: ...si podemos examinarnos a nosotros mismos, con nuestros colegas, saber qué hacemos bien y qué no hacemos bien, entonces podemos mejorar constantemente, inclusive sobresalir institucionalmente... (L-3); por lo mismo, coincidiendo con Ortega Ruíz (2014), la autorreflexión es "mirarse a sí mismo" o a sí misma, determinar aciertos, áreas por mejorar, establecer soluciones, una estrategia para promover la reestructuración de significados y un aprendizaje transformacional entre el personal docente de EF.

\section{Creencias y actuación pedagógica}

A continuación, se presentan los relatos escritos por el personal docente de educación física, referidos a sus prácticas pedagógicas. Se solicitó que estas experiencias tuvieran una estructura narrativa, referidas a tópicos específicos de su área de enseñanza, que permitan constatar el conocimiento curricular, del contenido, creencias sobre la enseñanza-aprendizaje, 
http://doi.org/10.15359/ree.25-2.9

conocimientos del contexto, recursos, metas y propósitos o productos a concretar. Estos relatos presentan dos partes: en la primera, narran una particular experiencia pedagógica vivida durante el desempeño de la profesión y, en la segunda, contestan a las siguientes interrogantes:

- ¿En qué consistió el problema o desafío que afrontaste en dicha práctica?

- ¿Qué decisiones pedagógicas tomaste para resolver el problema o desafío?

- ¿Qué conocimientos, creencias o teorías pedagógicas influyeron en las decisiones que tomaste?

- ¿Qué estrategias utilizaste?

- ¿Cuáles fueron las consecuencias de las decisiones pedagógicas tomadas?

\section{Caso 1:}

-En el área de Educación Física dentro de una de mis sesiones de aprendizaje se les solicitó materiales como conos, sogas, aros y las acciones desarrolladas fueron tan divertidas y diversificadas dado que el cincuenta por ciento del proceso fueron planificados por mi persona y el otro cincuenta por ciento fueron ideas espontáneas o creatividad de los mismos alumnos y lo más rescatable es que los estudiantes en cada una de las siguientes sesiones lo han manifestado con mayores entusiasmos y acciones con buenas actividades creativas positivas y los estudiantes al final se han sentido contentos y satisfechos.

- ¿En qué consistió el problema o desafío que afrontaste en dicha práctica?

- En toda sesión de aprendizaje no todos los estudiantes cumplen con traer sus materiales, sucede que los estudiantes han tenido que compartir con sus compañeros.

- ¿Qué decisiones pedagógicas tomaste para resolver el problema o desafío?

- La decisión que se ha tomado era que los estudiantes que no tenían o contaban con material educativo compartieran con sus compañeros.

- ¿Qué conocimientos, creencias o teorías pedagógicas influyeron en las decisiones que tomaste?

- La teoría de Novak conocimiento y aprendizaje.

- ¿Qué estrategias utilizaste? 
http://doi.org/10.15359/ree.25-2.9

http://www.una.ac.cr/educare

educare@una.ac.cr

- Mando directo y descubrimiento guiado.

- ¿Cuáles fueron las consecuencias de las decisiones pedagógicas tomadas?

- La participación conjunta de los estudiantes para un aprendizaje (L2).

\section{Caso 2:}

- Alrededor de la piscina, los estudiantes se mojan las manos, la cara, los pies, luego se sumergen a la piscina agarrándose de la canaleta para poder caminar en la parte más baja de la piscina.

Ya familiarizados con el agua en grupos de 5 en fila tomados de la cintura caminan en la piscina a una altura de 1 metro para luego tomados de las manos se mojan y a la voz del profesor se sumergen hasta mojarse la cabeza.

- ¿En qué consistió el problema o desafío que afrontaste en dicha práctica?

- Los estudiantes tienen temor al agua

- ¿Qué decisiones pedagógicas tomaste para resolver el problema o desafío?

- Busqué confianza a través del juego a fin de crear confianza al agua ya que esto permite aprender más rápido y tenga seguridad para sus posteriores aprendizajes

- ¿Qué conocimientos, creencias o teorías pedagógicas influyeron en las decisiones que tomaste?

- Las relaciones sociales y la familiarización con el agua en el entorno de la práctica de la natación.

- ¿Qué estrategias utilizaste?

- Mando directo y descubrimiento guiado

- ¿Cuáles fueron las consecuencias de las decisiones pedagógicas tomadas?

- Se logró el aprendizaje de la natación por la totalidad de los estudiantes del salón. (P2) 
http://doi.org/10.15359/ree.25-2.9

\section{Caso 3:}

En la ejecución práctica de la elaboración del fixture mediante el sistema de Campeonato Número fijo Antihorario debido a que los estudiantes aprendieron por observación solamente y no practicaron la elaboración del fixture con la participación variada de equipos, por lo tanto, cuando se enfrentaron a la realidad trayendo consigo desazón e impotencia.

La decisión pedagógica tomada es realizar inmediatamente una Retroalimentación a fin de que su aprendizaje implique un cambio que perdure en el tiempo se practica con participación de variedad de equipos participantes a través del ensayo con diferente número de participantes.

En nuestra decisión influyeron las relaciones sociales y la práctica con la participación de diferente cantidad de equipos.

Se utilizó las estrategias del ensayo - descubrimiento guiado, trabajo colaborativo, organización y planificación

Como consecuencia se consiguió autoeficacia en situaciones de logro en el espacio o ámbito socio constructivista que permitieron lograr un mejor desenvolvimiento en la práctica reduciendo el tiempo y mayor eficacia, seguridad y exactitud en la elaboración de fixture. (L1)

\section{Caso 4:}

Un alumno del semestre 2018-Il en el curso de Investigación, cuando estoy presentando el curso, se levanta y me pide a "boca de jarro" que defina el concepto de investigación acción (¿para usted que es investigación acción?), de eso se trata, vamos a conocer no solo el concepto y sus características y utilidades le respondo, pero el insiste en su pedido, ensayé una definición, quedó un tanto descontento eso es lo que noté, le pedí que prestara atención a las actividades programadas para la sesión.

En efecto, en la sesión sobre el concepto, y las características de la investigación acción en el momento de la motivación interrumpe el alumno, no deja desarrollar la sesión anunciada, obviamente, cambio toda la planificación que había considerado, me vi obligado a aperturar el diálogo con el alumno, solicité explicara lo que deseaba manifestar, lo hizo y se continuó con la sesión, pero tuve que cambiar la estrategia y procedí a exponer y busqué la participación dialógica de toda la sala, la participación no fue total. (P1) 
http://doi.org/10.15359/ree.25-2.9

http://www.una.ac.cr/educare

educare@una.ac.cr

Estas narraciones nos permiten acercarnos a la problemática de la subjetividad docente a través de una caracterización de las prácticas pedagógicas en el área de educación física, se advierte que predominan las prácticas pedagógicas convencionales, más técnicas e instruccionales, que procesos de acción-reflexión de indagación o experimentación que, por lo general, desembocan en la perspectiva pedagógica de la asimilación y adquisición de conocimientos, acompañado, según Rivera Sosa et al. (2020), de algunos atributos parciales y apenas explicativos en contraste con los referentes teóricos y orientaciones pedagógicas actuales de la EF. Situación que, a decir de Betancur-Agudelo et al. (2018), genera una mínima relación entre las prácticas pedagógicas de $\mathrm{EF}$, los planes institucionales, el contexto y los propósitos del área curricular.

Según los resultados ofrecidos en los cuatro casos presentados, la concatenación entre los saberes experienciales (trayectoria pedagógica personal), saberes de la enseñanza (conocimientos base de los procesos de enseñanza-aprendizaje) y los saberes pedagógicos, que se expresan en las actividades y estrategias concretas de aprendizaje son poco evidenciables, se hace difuso distinguir los procedimientos seguidos entre las didácticas especiales y la general. A este respecto, manifiesta Quijano López (2015, p. 60) que "las didácticas específicas se desarrollan mediante la complementariedad de dos matices: el primero de ellos es el de los contenidos acumulados propios de la especificidad, y el segundo se corresponde con los procedimientos que permiten la construcción de nuevos conocimientos", por consiguiente, debe propenderse a una relación no solo mediacional, sino de cooperación constructiva, recíproca entre las didácticas específicas y la general.

En el caso 3 de las narrativas presentadas, el docente, luego de ensayar de manera empírica las actividades de aprendizaje, reconoce que ...fue un absoluto fracaso tanto para estudiantes como para el profesor...(L1), es un hecho que denota la fragilidad por la concepción que el docente y la docente tienen respecto de lo que es aprender y del tacto que se posee como profesional de la enseñanza. El tacto en la enseñanza, según manifiesta van Manen (1998, p. 139), se refiere a "una relación humana íntima, a la intimidad, a la conexión. Que un profesor 'haya conectado', que esté 'en íntimo contacto' con los alumnos, significa que sus acciones están determinadas por una sensibilidad con tacto". Dada esta situación, surgen dos tipos de consecuencias, a decir de Sánchez-Sánchez y Jara-Amigo (2019):

En primer lugar, el reconocimiento de un trabajo docente constituido por tensiones y dilemas, pues su relación con el alumnado está constituida fundamentalmente por relaciones sociales, que obligan al despliegue de negociaciones e interacciones. En segundo lugar, la ausencia de un control total ejercido por docentes sobre estudiantes, lo que dificulta el trabajo y el logro de objetivos en la práctica. (p. 18) 
http://doi.org/10.15359/ree.25-2.9

Independientemente de la concepción pedagógica o teórica que, de manera explícita, enuncia el personal docente de educación física, es usual que el proceso de enseñanzaaprendizaje que llevan a cabo en su práctica sea diferente a lo que señalan de manera verbal, como ocurre en el caso 1. A nivel de enunciación, algunos maestros o maestras ostentan conocimientos teórico-pedagógicos, hasta logran conceptuar y fundamentar ciertos enfoques y teorías referidas a enseñanza-aprendizaje, pero finalmente, no se advierte que los pongan en práctica en su desempeño profesional. Los modelos predominantes de enseñanza de la educación física son, básicamente, la clase magistral, el mando directo y el uso de los textos de enseñanza: Según el sílabo del componente curricular a mi cargo, generalmente los estudiantes investigan información en los textos proporcionados... (P-1). Se puede inferir, entonces, que docentes de EF requieren renovar su actuación pedagógica, adoptar nuevos marcos teóricos y prácticas más eficaces, contextualizadas y flexibles.

Es más, luego de veinte años de iniciarse la aplicación del enfoque por competencias, señala Díaz Díaz (2015), "la mayoría de profesores continúa teniendo dificultades para programarlas y evaluar, así como para manejar los aprendizajes transversales y las nuevas tecnologías aplicadas a las áreas de formación" (p. 43). En efecto, en los casos presentados, es notorio que en los procesos de enseñanza-aprendizaje de la educación física se priorizan los protocolos de ejecución eficaz del rendimiento físico dispuesto por el personal docente, antes que la construcción de aprendizajes a partir de saberes previos y de manera significativa, situación que puede provocar en el estudiantado una desmotivación frente a la actividad física y la desvalorización de la asignatura de Educación Física.

Es una constante en el discurso de estos relatos respecto a la praxis educativa advertir que la prioridad en la relación educativa, generalmente, está conferida al personal docente; lo que dice y hace es el parámetro para juzgar como bueno o malo de lo que se hace en la clase, es quien tiene la última palabra y lo sabe todo, situación o estrategias que pueden conllevar a la asunción de un rol docente autoritario, que provoca un desencuentro con sus estudiantes, al privilegiar la enseñanza y devaluar el aprendizaje y el rol del estudiantado. Se debe tomar en cuenta que las interacciones dialógicas y afectivas centradas en procesos subjetivos tienen una incidencia muy significativa en buena parte de las dinámicas de aprendizaje y enseñanza.

Se asume por relación educativa, el proceso de interacción entre estudiantes que construye significados, componente curricular o asignatura por aprender y docentes que asumen el papel mediador entre conocimientos y aprendizajes. A estos tres elementos Coll (1997) los denomina triángulo interactivo. En los casos presentados, la relación docente-estudiante es más de dominio, sumisión y pasividad, interacciones que, por lo general, se advierten en los momentos de inicio y ejecución de los desempeños físicos deseados, según los protocolos y disciplina establecidos por el personal docente, hechos con los que resignifica subjetivamente en el aula los vínculos de dependencia. En estas circunstancias, es preciso construir y resignificar la función docente en la educación física, por cuanto la identidad y la representación que tiene el profesorado de sí mismo como profesional de la enseñanza está en riesgo. 
http://doi.org/10.15359/ree.25-2.9

http://www.una.ac.cr/educare

educare@una.ac.cr

En este escenario, la experiencia acumulada y los conocimientos que dominan por años (casi invariablemente), constituyen para docentes de educación física, por lo general, las únicas herramientas con los que pretende tener éxito en aula: Nosotros no hemos tenido oportunidades para recibir capacitación o actualizaciones, como los han tenido los docentes de educación básica regular. En los pedagógicos por años no hubo nombramiento de nuevo personal docente, hará como más de 15 años, como tampoco hemos recibido en estos años, cursos como éste. Recién ahora, se han acordado de nosotros, pero dicen que es para evaluarnos y seguro nos sacarán con ese pretexto (L4); Cumplí más de 25 años de servicio como docente de Educación Física, estoy por irme y recién ahora el Ministerio nos ofrece un programa de fortalecimiento de capacidades... (L3). En efecto, en las últimas décadas, no se registran, en los medios oficiales del Estado peruano, acciones y políticas educativas específicas, orientadas a cubrir la formación en servicio del personal docente de educación física en los institutos superiores pedagógicos. La acción más notoria emprendida como política de Estado para el personal docente de educación física, corresponde al Plan de Fortalecimiento de la Educación Física y el Deporte Escolar, implementada durante el 2016, con carácter experimental para docentes de educación básica regular, únicamente (Maquera Maquera y Bermejo Paredes, 2017). Por su parte, el personal docente de la EPEF-UNA Puno, cubre, generalmente, los procesos de formación en servicio, de manera autogestionaria: Cada quién tiene su maestría o doctorado, por esfuerzo propio... (P3).

De este modo, estamos ante una formación continua de docentes de educación física, cualitativamente desatendida y postergada por el Estado peruano, situación propensa a convertirse en un problema estructural de la educación superior. Ninguna acción o política educativa puede tener éxito sin considerar el factor docente. Es más, en el acto didáctico, el componente docente desempeña un papel clave, cumple una función mediadora entre el proyecto diseñado y las prácticas docentes, el profesorado como agente curricular y no como ejecutor mecánico, trasladará el currículo a la práctica no solo mediatizado por el contexto escolar, sino por su manera propia y personal de entender la materia objetivo de enseñanza (Bolívar Botía, 2015). A este respecto, Correa-Díaz et al. (2019) precisan que: "son las personas, más que las tecnologías y los procesos, las agentes de cambio y las generadoras de innovación" (p. 17).

\section{Afrontamiento de la diversidad cultural}

Puno es la única región del Perú donde subsisten las dos culturas prehispánicas de mayor trascendencia en la cordillera de los Andes: el aimara y el quechua. Según los Censos Nacionales 2017: XII de población, VII de vivienda y III de comunidades, alrededor del 70\% se autodefine como aimara-quechua (Instituto Nacional de Estadística e Informática [INEI], 2018), otros datos grafican a Puno como uno de los lugares del país con mayores problemas educativos por resolver: "solo dos de cada diez niños en Puno comprendían lo que leían" (Grupo Propuesta Ciudadana, 2016, p. 6), y la presencia manifiesta de la discriminación racista por el origen cultural quechua o aimara presente en las escuelas (Cueto et al., 2010), factores directamente asociados a la realidad de educación en Puno. 
http://doi.org/10.15359/ree.25-2.9

Si se concibe que educar en un contexto de diversidad cultural es rechazar cualquier tipo de exclusión educativa, entones, está implícito que, en Puno, deben resarcirse deudas históricas pendientes con la educación de su población mayoritariamente de raíz indígena aimara-quechua. En pleno siglo XXI, la formación inicial de docentes, tanto en la Universidad Nacional del Altiplano (UNA) como en el Instituto Superior Pedagógico Público de Educación Física de Lampa (ISPPEF), Puno-Perú, carece de propuestas curriculares y de políticas educativas específicas de inclusión, según las particularidades de sus estudiantes y el contexto de diversidad cultural predominante. Conforme señalan Chen-Quesada y Salas-Soto (2018):

Los planes de estudio deben responder a estudios minuciosos y que puedan ser revisados a la luz de nuevas tendencias curriculares y otras investigaciones interdisciplinarias y transdisciplinarias que nutran la teoría y la práctica. Por esta razón, la universidad no debe ser un ente aislado de su realidad social sino; por lo contrario, tener una amplia participación a fin de comprender de cerca las dinámicas que confluyen y sus implicaciones sobre la realidad social. (p. 28)

La malla curricular establecida en 10 semestres académicos, para la formación inicial docente en ambas instituciones estudiadas, registra, coincidentemente, apenas una sola asignatura relacionada con la actuación docente en contextos de diversidad cultural. En la EPEF, recibe la denominación de realidad nacional y educación intercultural consignada en el IV semestre académico (Escuela Profesional de Educación Física [EPEF], 2019) y, en la ISPPEF Lampa, está identificada como educación intercultural, para el III semestre académico (Instituto Superior Pedagógico Público Educación Física ISPPEF, 2017). Por consiguiente, la formación inicial pedagógica en estas dos instituciones de educación superior debe nutrirse del tratamiento de la singularidad y la excepcionalidad, como rasgos inherentes a toda persona y entorno inmediato.

No solo el sistema homogeneizante, que muchas veces limita y condiciona la autonomía y el ser profesional de la educación, juega en contra de la educación en contextos mediados por la diversidad cultural, sino en nuestro medio, la circulación de subjetividades enraizadas en la colonialidad del poder, surten efectos distintos: ... me excluyeron de la convocatoria a nombramiento docente 2018 en la universidad, por no tener el grado de magíster en la especialidad de Educación Física. Pero, tengo una maestría en el área y es de educación intercultural. Esto fue arbitrario y discriminatorio para mí. La Ley Universitaria 30220 no exige especializaciones específicas para ejercer la docencia universitaria, es suficiente contar con el grado de magíster, únicamente (P2). Además, si tomamos en cuenta la misión y visión de ambas instancias, hallaremos que existen grandes distancias entre lo que se hace y lo que se pretende ser o hacer; no están directamente comprometidas y asociadas a la problemática educativa, social, cultural y política de un contexto excluyente y de diversidad cultural. Desde la posición cultural, se conoce que el 
http://doi.org/10.15359/ree.25-2.9

http://www.una.ac.cr/educare

educare@una.ac.cr

aimara no desea la escuela para un posible bienestar en el futuro, sino la educación y la escuela deben permitirle el bienestar hoy mismo y por siempre (Bermejo-Paredes y Maquera-Maquera, 2019). Por consiguiente, reivindicar el carácter histórico, situacional del ser humano, se hace irrenunciable, es fundamental "partir de presupuestos éticos distintos (otro paradigma) que haga posible una educación que empiece por el otro, que se pregunte por el otro en toda su realidad" (Ortega Ruíz, 2014, p. 76); es condición sine qua nom, constituir espacios de reflexión que permitan pensar y argumentar éticamente para viabilizar nuestras propuestas, asumiendo la realidad, la existencia humana y el ejercicio del pensamiento como lugares de encuentro y de interrelación, manteniendo el principio de la dignidad de todas las personas.

\section{Conclusiones}

El análisis de contenido practicado a las entrevistas y narraciones construidas por los sujetos participantes del estudio permite comprender que la subjetividad del personal docente de educación física tanto en el ISPPEF-Lampa como en la EPEF-UNA-Puno, está representada por los sentidos otorgados a la tarea de la enseñanza, la expresión de sus emociones y actitudes, donde resalta, en primer plano, que las cualidades de actuación como profesional de la enseñanza en EF están en riesgo, a partir de la ruptura entre lo que se es y lo que se hace, y al no evidenciarse mayores explicaciones del "por qué actúo como lo hago" en el desarrollo de las prácticas pedagógicas de EF; no obstante, los resultados no son suficientes para caracterizar las actuaciones del personal docente de EF. Se requiere incidir en la reconstrucción sistemática de su conocimiento experiencial; en los estudios de análisis autorreflexivo de su propia identidad, comprensión intelectual y profesional, teniendo en consideración el sistema histórico y el contexto de diversidad cultural en el que se vive y actúa, sin aislar lo individual de lo social; de relacionar las partes con el todo y el todo con las partes, desde la cotidianidad de la enseñanza-aprendizaje, el reconocimiento de una pluralidad de construcciones de la realidad, representaciones, concepciones y creencias.

De acuerdo con las representaciones subjetivas de docentes de EF, se le atribuye a los procesos de autorreflexión ser uno de los principales componentes motivacionales para evaluar, tomar decisiones, pensar autónomamente y actuar a favor de prácticas pedagógicas inclusivas y de transformación; en cambio, la actuaciones pedagógicas están signadas por la fragilidad de concatenación, entre los saberes experienciales (trayectoria pedagógica personal), saberes de la enseñanza (conocimientos base de los procesos de enseñanza-aprendizaje) y los saberes pedagógicos no son claramente evidenciables, situación que se justifica emotivamente, culpando al Estado por su olvido del personal docente de educación física. Por otra parte, es preciso señalar que el contexto cultural predominantemente indígena de la región Puno no forma parte de las prácticas pedagógicas y programas de estudio para la formación docente inicial en EF, hecho a partir del cual es posible sostener que la EF está pensada para contextos 
http://doi.org/10.15359/ree.25-2.9

inexistentes e individuos sin rostro. Por consiguiente, tanto docentes de EF así como las instituciones formadoras de docentes en EF deben replantear sus prácticas pedagógicas y políticas de intervención de manera que incidan en lo que ocurre en una clase de EF y a su vez se proyecten fuera de los recintos institucionales, se elaboren nuevos discursos pedagógicos, se reconozcan las nuevas formas de subjetividad y el valor del ejercicio profesional autónomo y emancipador del personal docente que reflexiona críticamente para mejorar la calidad de los procesos de enseñanza-aprendizaje, se involucra en el contexto y va cerrando las brechas entre los modelos académicos y las estructuras organizacionales convencionales.

El personal docente de EF cuenta con un saber experiencial sustentado en representaciones o significaciones forjadas en su trabajo, estrechamente relacionado con el sentido de sus actuaciones pedagógicas, asunto que debe integrarse en un proceso continuo a los saberes provenientes de las teorías o enfoques pedagógicos, a través de planes o programas constituidos en herramientas de ejercicio reflexivo que tomen en cuenta las significaciones o procedimientos que dotan de sentido de realidad a los sujetos, sobre lo que se hace y dónde se hace, para mejorar e innovar la teoría y práctica de los procesos de enseñanza-aprendizaje, la relación entre estructuras institucionales y sujetos, así como, el desarrollo y formación docente auténticos de educación física.

\section{Declaración de Material complementario}

Este artículo tiene disponible, como material complementario:

-La versión preprint del artículo en https://doi.org/10.5281/zenodo.39223234

\section{Referencias}

Andréu Abela, J. (2002). Las técnicas de análisis de contenido: Una revisión actualizada. Fundación Centro de Estudios Andaluces.

Bermejo-Paredes, S. y Maquera-Maquera, Y. A. (2019). Interpretación de la escuela rural andina en comunidades aimaras de Puno-Perú. Revista Electrónica Educare, 23(2), 1-15. https:// doi.org/10.15359/ree.23-2.4

Betancur-Agudelo, J. E., López-Ávila, C. R.y Arcila-Rodríguez, W.O. (2018). El docente de educación física y sus prácticas pedagógicas. Revista Latinoamericana de Estudios Educativos, 14(1), 15-32. http://190.15.17.25/latinoamericana/downloads/Latinoamericana14(1)_2.pdf

Bolívar Botía, A. (2015). Didáctica y currículum: Campos disciplinares. En J. D. Segovia y M. Pérez Ferra (Coords.), Aprendiendo a enseñar. Manual práctico de didáctica (pp. 21-34). Pirámide. 
http://doi.org/10.15359/ree.25-2.9

http://www.una.ac.cr/educare

educare@una.ac.cr

Bolívar Botía, A., Segovia, J. D. y Fernández Cruz, M. (2001). La investigación biográfico-narrativa en educación. Enfoque y metodología. Muralla.

Castellanos Galindo, S. H. y Yaya Escobar, R. E. (2013). La reflexión docente y la construcción de conocimiento: Una experiencia desde la práctica. Sinéctica Revista Electrónica de Educación, 41, 1-18. https://www.redalyc.org/pdf/998/99828325005.pdf

Cerdá Michael, A. D: (2001). Nosotros los maestros. Concepciones de los docentes sobre su quehacer. Universidad Pedagógica Nacional.

Chen-Quesada, E. y Salas-Soto, S. (2018). Referentes curriculares para la toma de decisiones en materia de planes de estudio de educación superior. Revista Electrónica Educare, 23(3), 1-31. https://doi.org/10.15359/ree.23-3.7

Coll, C. (1997). ¿Qué es el constructivismo? Magisterio del Río de la Plata.

Corbetta, G. (2003). Metodología y técnicas de investigación social. McGrawHill.

Correa-Díaz, A. M., Benjumea-Arias, M. y Valencia-Arias, A. (2019). La gestión del conocimiento: Una alternativa para la solución de problemas educacionales. Revista Electrónica Educare, 23(2), 1-27. https://doi.org/10.15359/ree.23-2.1

Cueto, S., Guerrero, G., León, J., Zevallos, Á. y Sugimaru, C. (2010). De quinto de primaria al fin de la secundaria en seis años: Un estudio longitudinal en Puno (Doumento de trabajo 6). GRADE; CIES. www.grade.org.pe/wp-content/uploads/ddt56.pdf

Díaz Díaz, H. (2015). Formación docente en el Perú. Realidad y tendencias. Santillana.

Escuela Profesional de Educación Física. (2019). Plan de estudios. Facultad de Ciencias de la Educación, Universidad Nacional del Altiplano. portal.unap.edu.pe/?q=educacionfisica

Flick, U. (2007). Introducción a la investigación cualitativa. Morata.

González Rey, F. (2002). Sujeto y subjetividad: Una aproximación histórico cultural. Ediciones Paraninfo.

Grupo Propuesta Ciudadana. (2016). Gestión estratégica de la educación en Puno: El currículo y la identidad como ejes de cambio. Autor. http://repositorio.minedu.gob.pe/ handle/123456789/4685

Hernández-Álvarez, J. L., Velázquez-Buendía, R., Martínez-Gorroño, M. E., y Díaz del Cueto, M. (2010). Creencias y perspectivas docentes sobre objetivos curriculares y factores determinantes de actividad física. Revista Internacional de Medicina y Ciencias de la Actividad Física y El Deporte, 10(38), 336-355. http://cdeporte.rediris.es/revista/revista38/ artcreencias160b.pdf 
http://doi.org/10.15359/ree.25-2.9

Instituto Nacional de Estadística e Informática. (2018). Perú: Perfil sociodemográfico. Informe Nacional. Censos Nacionales 2917: XII de población, VII de vivienda y III de comunidades indígenas. Autor. https://www.inei.gob.pe/media/MenuRecursivo/publicaciones digitales/Est/Lib1539/libro.pdf

Instituto Superior Pedagógico Público Educación Física. (2017). Plan de estudios [Blog]. http:// iesppeflampa.blogspot.com/p/plan-de-estudios.html

Krippendorff, K. (1990). Metodología del análisis de contenido. Teoría y práctica. Paidós.

Maquera Maquera, Y. A. y Bermejo Paredes, S. (2017). Indicadores de capacidades físicas en estudiantes de educación secundaria desarrollados por el plan de fortalecimiento de la educación física y el deporte escolar Puno 2016. Revista de Investigaciones Altoandinas, 19(1), 75-84. https://doi.org/10.18271/ria.2017.257

Martínez, M. (1999). Comportamiento humano. Nuevos métodos de investigación. Trillas.

Ortega Ruíz, P. (2014). La pedagogía de la alteridad como paradigma de la educación intercultural. En P. Ortega Ruíz (Coord.), Educar en la alteridad (Tomo I, pp. 55-80). Editorial Redipe.

Patiño-Garzón, L. y Rojas-Betancur, M. (2009). Subjetividad y subjetivación de las prácticas pedagógicas en la universidad. Educación y Educadores, 12(1), 93-105. https://dialnet. unirioja.es/servlet/articulo?codigo $=2983797$

Quijano, A. (2000). Colonialidad del poder, eurocentrismo y América Latina. En E. Lander (Comp.), La colonialidad del saber: Eurocentrismo y ciencias sociales. Perspectivas latinoamericanas (pp. 201-246). CLACSO.

Quijano López, R. (2015). Didáctica general y didácticas específicas: Qué las une y qué las diferencia En J. D. Segovia y M. Pérez-Ferra (Coords.), Aprendiendo a enseñar. Manual práctico de didáctica (pp. 59-66). Pirámide.

Rivera Sosa, J. M., Arras Vota, A. M. de G., Tarango, J., Mendoza Meraz, G., y López Alonso, S. J. (2020). Educación física: Referentes y nociones conceptuales del personal docente en Educación Primaria. Retos, 39, 298-305. https://doi.org/10.47197/retos.v0i39.58114

Sánchez-Sánchez, G. I. y Jara-Amigo, X. E. (2019). Estudiantes, docentes y contexto educativo en la representación del profesorado en formación. Revista Electrónica Educare, 23(3), 1-21. https://doi.org/10.15359/ree.23-3.8

van Manen, M. (1998). El tacto en la enseñanza. El significado de la sensibilidad pedagógica. Paidós Educador. 